\title{
Uso de indicadores da qualidade para avaliação de prestadores de serviços públicos de odontologia: um estudo de caso*
}

\author{
Schelle Aldrei de Lima Da Soller** \\ Gilsée Ivan Regis Filho***
}

Sumário: 1. Introdução; 2. Metodologia; 3. Resultados e discussão; 4. Conclusões.

Summary: 1. Introduction; 2. Methodology; 3. Results and discussion; 4. Conclusions.

Palavras-chave: indicadores; qualidade; odontologia.

KEYWORDs: indicators; quality; dentistry.

Qualidade é um tema muito discutido, tendo em conta a grande concorrência, a necessidade de conter custos e a maior exigência dos clientes. Exigência esta que se estende a todos os tipos de serviços, inclusive os serviços de saúde. No entanto, não basta apenas dizer que se presta um atendimento de qualidade; é preciso avaliá-la e esta avaliação pode ser realizada pelo uso dos Indicadores da Qualidade, que em saúde são categorizados em três grupos: Estrutura, Processo e Resultado. Desta forma, este trabalho se propôs avaliar um serviço público de odontologia com a utilização de um Indicador da Qualidade de cada categoria. Sendo "horas de treinamento ou cursos fornecidos pela instituição/cirurgião-dentista/ano" o Indicador de Estrutura selecionado, "cuidados com biossegurança" o de Processo e "satisfação do prestador" o de Resultado. Conclui-se que os indicadores aplicados são excelentes instrumentos de avaliação e fonte de dados para planejamento de um serviço de odontologia.

\footnotetext{
* Artigo recebido em jul. 2010 e aceito em dez. 2011.

** Mestre em odontologia em saúde coletiva do Programa de Pós-Graduação em Odontologia da Universidade Federal de Santa Catarina (UFSC). Cirurgiã-dentista. Endereço: Av. Othon Gama D’Eça, 900, sala 601 - Centro - CEP 88015-240, Florianópolis, SC, Brasil. E-mail: Schelle_soller@hotmail.com.

*** Doutor em engenharia da produção, mestre em ergonomia, especialista em desenvolvimento de recursos humanos, especialista em saúde pública, bacharel em administração. Professor associado I, aposentado, da UFSC. Endereço: Rua 700, 160, ap. 403 - Centro - CEP 88330-618, Balneário Camboriú, SC, Brasil. E-mail: gregis@brturbo.com.br.
} 


\begin{abstract}
Use of indicators for quality in health's publics services of dentistry: a case report

Quality is a theme very discussed, given the great competition, the need to contain costs and the customers' largest demand. Demand this that extends the all the types of services, besides the services of health. However, to say that a quality attendance is rendered is not enough it is just necessary to evaluate her and this evaluation can be accomplished by the use of the Indicators of the Quality, that you/they are classified in three groups in health: He/she/you structures, Process and Result. This way, this work intended to select and to test an Indicator of the Quality of each category in a public service of dentistry with the objective of verifying your execution. Being "hours of training or courses supplied by the institution/surgeon dentist/year" the Indicator of Structure selected, "cares with biosafety" the one of Process and "satisfaction of the professional" the one of Result. It is ended that the applied indicators are excellent evaluation instruments and source of data for planning of a service of dentistry.
\end{abstract}

\title{
1. Introdução
}

A literatura científica apresenta de forma significativa a importância da gestão pela qualidade tanto para o setor de produção quanto para o de operações de serviços, seja em empresas privadas ou públicas, e como se deve atuar para alcançar a excelência nesses setores.

Por outro lado, a busca pela qualidade deve ocorrer, também, nos serviços públicos de saúde, onde a sociedade está exigindo de seus gestores excelência nos serviços a ela prestados. Entretanto, essa exigência torna fundamental a criação de normas e mecanismos de avaliação e controle da qualidade neste setor (Kluck et al., 2002). Assim, é necessário medir constantemente os resultados para que se obtenham dados precisos e concretos da atual situação e do que deve, prioritariamente, ser melhorado, tornando-se importante demonstrar fatos, conceituar produtividade em saúde e embasar as reivindicações de melhoria contínua da qualidade (Nepote, 2003).

Fadel e Regis Filho (2009) observaram que, para os clientes, os aspectos mais significativos na percepção da qualidade em serviços públicos de odontologia, da Prefeitura Municipal de Florianópolis, são a tranquilidade ao utilizar os serviços e a clareza ao esclarecer dúvidas (91\%), e para os cirurgiões-dentistas é a boa execução dos serviços (100\%). Tal fato está de acordo com o encontrado na literatura, pois para os profissionais de odontologia a qualidade técnica dos serviços é altamente significativa e para os clientes a qualidade interpessoal é mais significativa. De maneira geral, houve concordância nas respostas de ambos os grupos estudados. 
Dados obtidos por Milan, Toni e Barazetti (2005) ressaltam que tão importante quanto identificar as imagens de serviços em relação a um público-alvo específico é entender como as imagens estão configuradas. A partir da revisão da literatura, observou-se que a imagem de serviços é um conjunto de representações, impressões, convicções e redes de significados de um objeto, ou seja, o serviço é armazenado na memória de forma holística, podendo ser configurado a partir de um conjunto de atributos funcionais, simbólicos, cognitivos e emocionais, organizados em torno de alguns elementos centrais e/ou periféricos.

A identificação dos atributos e seus elementos constituintes revela que as imagens estão muito relacionadas às expectativas e às características de cada tipo de paciente. Informações deste tipo são importantes para a gestão dos serviços, pois para cada público podem ser direcionadas ações de acordo com suas expectativas e percepções.

Para Tengan (2008), a avaliação da qualidade em serviços de saúde constitui um passo importante no planejamento e na gestão dos serviços. O autor utilizou como ferramenta para planejar a qualidade em um serviço público odontológico um método de qualidade denominado Quality Function Deployment (QFD - Desdobramento da Função Qualidade). Após a análise dos dados e a construção da matriz da qualidade, o autor verificou que a porcentagem de usuários satisfeitos, o número de reclamações/mês, o número de tratamentos completados/mês e o número de solicitações para manutenção dos equipamentos e periféricos foram as características de qualidade mais importantes para atender aos usuários. Os resultados do trabalho demonstraram que o QFD pode ser aplicado no serviço público odontológico, constituindo uma ferramenta de qualidade importante e viável na avaliação e no planejamento das ações em saúde bucal, uma vez que, por meio dessa metodologia, é possível entender e atender ao usuário, reconhecendo os fatores que se traduzem em qualidade.

Por outro lado, Miguel e Salomi (2004) afirmam que se faz necessário ressaltar que a revisão da literatura levanta algumas questões em relação à medição da qualidade em serviços externos e que essas questões não foram resolvidas por completo pelos pesquisadores, sugerindo uma falta de consenso. Citam como exemplo a relação de causalidade entre a qualidade dos serviços e a satisfação de clientes e a influência do preço na satisfação do cliente.

Outro aspecto é a generalização das dimensões para todos os tipos de serviços, pois ainda há controvérsias quanto à generalização das cinco dimensões da qualidade e a influência de cada uma dessas dimensões na percepção de qualidade, considerando tipos diversos de serviços. 
Segundo Tontini e Sant'Ana (2008), atender as necessidades dos clientes é peça-chave para sua satisfação e para o sucesso de uma organização no longo prazo, pois clientes satisfeitos tendem a repetir o consumo com a organização e são menos sensíveis a preços. $\mathrm{O}$ atendimento dessas necessidades depende do desempenho do produto ou do serviço em seus diversos atributos, pois um projeto superior, que inclua os atributos relevantes para o consumidor, pode trazer grande diferencial no mercado para a organização. Assim, a identificação da relação entre o desempenho dos atributos e a satisfação do consumidor torna-se crítica para o sucesso em um mercado competitivo.

Tontini e Silveira (2007) afirmam que quanto maior o desempenho maior a satisfação e vice-versa. Para os autores, atributos atrativos são aqueles cuja presença ou desempenho elevado traz satisfação superior, porém sua ausência ou desempenho inferior não causam insatisfação. Ignorar este relacionamento não linear entre o desempenho dos atributos e a satisfação geral do cliente pode levar a decisões errôneas na hora de se identificar quais atributos são críticos e quais ações devem ser tomadas para aumentar a satisfação dos clientes. Entre estas decisões está melhorar um atributo básico acima do nível médio do mercado. Atributos básicos não trarão satisfação superior se a empresa tiver desempenho acima dos concorrentes e acima da média do mercado. Neste caso, o esforço de melhoria seria desnecessário.

Entretanto, introduzir um atributo atrativo e de alto custo, em virtude de se estar com desempenho inferior aos concorrentes, também pode ser um erro, pois um desempenho inferior nestes atributos não traz insatisfação aos clientes. Neste caso, a empresa poderia estar desviando recursos de outras áreas mais relevantes.

De acordo com Valenzuela (2005), qualquer planejamento em saúde deve se pautar em informações, sejam estas quantitativas ou qualitativas, que possibilitem o conhecimento da realidade para propor metas e objetivos. Segundo Soárez, Padovan e Ciconelli (2005), estas informações podem ser fornecidas através de Indicadores da Qualidade em Saúde, pois quando bem administrados esses indicadores constituem ferramenta fundamental, tanto para a gestão quanto para avaliação do sistema em particular e da saúde da população como um todo. Entretanto, para utilizar um indicador é importante garantir acesso a informações ou dados de boa qualidade, precisos, confiáveis e de forma regular (Soárez, Padovan e Ciconelli, 2005; Cecílio, 2002; Donabedian, 1988a).

Através dos resultados obtidos pelos indicadores é possível avaliar uma dada situação, realizar um planejamento adequado, definir melhorias necessárias, bem como obter informações que auxiliem as tomadas de decisão, 
a melhor alocação dos recursos, a gestão e, consequentemente, melhorar a qualidade da assistência oferecida aos usuários (Soárez, Padovan e Ciconelli, 2005; Marinho; 2001; Mello e Camargo, 1998).

Um bom indicador deve ter as seguintes características, segundo Bittar (2001):

1) Objetividade: deve ter um objetivo claro, expressando de forma simples e direta a situação a que se refere;

2) Simplicidade: fácil de calcular, de buscar os dados e analisá-los;

3) Validade: cumprir o propósito de identificar as situações que devem ser melhoradas;

4) Sensibilidade: o grau no qual o indicador é capaz de identificar todos os problemas existentes; e

5) Baixo custo: indicadores cujo valor financeiro é alto inviabilizam sua execução.

No setor saúde os indicadores podem avaliar o estado de saúde de uma população ou o desempenho dos serviços de saúde; a soma destas duas informações permite conhecer a situação sanitária de uma população (Valenzuela, 2005). Assim, quanto aos Indicadores da Qualidade capazes de avaliar os serviços de saúde, Donabedian, com muita propriedade, os agrupa em três categorias (Donabedian, 1988a):

1) Estrutura: avaliam os atributos dos locais nos quais o serviço é prestado, levando em consideração conveniência, conforto, silêncio, privacidade e assim por diante. Inclui recursos materiais (instalações, equipamentos e dinheiro), recursos humanos (número e qualificação dos profissionais) e recursos da estrutura organizacional (profissionais de saúde, métodos de reembolso);

2) Processo: avaliam as atividades de cuidados realizadas para com o paciente; são técnicas operacionais. Dividem-se entre o cuidado técnico propriamente dito (utilização dos conhecimentos científicos e tecnológicos da medicina) e a relação interpessoal entre paciente e profissional. Incluem as atividades do paciente buscando o cuidado, como também as atividades do médico dando o diagnóstico e realizando o tratamento; e

3) Resultado: avaliam os efeitos dos cuidados prestados anteriormente na saúde do paciente e da população e, também, o grau de satisfação do paciente e do prestador. 
Para Donabedian (1988a, 1988b), esta abordagem de três dimensões para avaliação da qualidade em serviços de saúde é possível porque boa estrutura aumenta a probabilidade de bom processo e bom processo aumenta a probabilidade de obter melhorias na saúde e bem-estar dos indivíduos ou populações, ou seja, bom resultado. Desta forma deve-se deduzir que condições estruturais podem ser tanto desfavoráveis quanto condutivas ao bom cuidado, não se podendo afirmar, entretanto, se o cuidado, em função destas, será bom ou ruim.

Estudo de Silveira, Santos e Costa (2001), realizado para avaliar a estrutura e o processo do atendimento pré-natal nas unidades de atenção primária, a saúde do município de Pelotas está de acordo com a afirmação de Donabedian (1988a, 1988b). Os autores classificaram a estrutura e o processo do cuidado, de grande parte das unidades de saúde, como precários, e um dos motivos citados como causa do cuidado precário é a dificuldade de acesso (estrutura), pelo fato de as unidades de saúde, na sua maioria, funcionarem apenas nos turnos diurnos. No entanto, esperam que serviços com uma melhor estrutura apresentem um processo de atendimento mais competente, porém essa condição não é essencial e dependente. Duarte e Ferreira (2006) também concordam com Donabedian (1988a, 1988b) quando afirmam que estrutura de qualidade não garante qualidade nos processos, assim como processos de qualidade não são garantia total de bons resultados. No entanto, indicam que bons resultados dificilmente serão obtidos com estrutura e/ou processo inadequados, ou seja, não garantem, porém influem.

Portanto, devido à complexidade de um serviço de saúde, um único indicador não é capaz de avaliar a qualidade do serviço prestado aos usuários, sendo necessário elencar um grupo de indicadores de cada categoria para este fim (Soárez, Padovan e Ciconelli, 2005; Malik e Schiesari, 1998) ou pelo menos um indicador de cada categoria.

O presente estudo tem como objetivo principal avaliar a qualidade dos serviços públicos de odontologia oferecidos por uma Secretaria Municipal de Saúde, através do uso de indicadores da qualidade e pela perspectiva do prestador ou cliente interno, bem como buscar os indicadores capazes de mensurar o desempenho dos serviços de saúde, não pretendendo avaliar o estado de saúde da população em questão. Como objetivos específicos busca:

1) Selecionar indicadores que se julguem adequados para serviços de odontologia;

2) Verificar se estes indicadores apresentam consistência, coerência e concordância para avaliar o serviço; 
3) Fornecer dados através da avaliação e dos resultados obtidos com os indicadores aos gestores que possam auxiliar no planejamento dos serviços;

4) Detectar possíveis falhas na prestação dos serviços para que possam ser reavaliados e melhorados; e

5) Apontar melhorias para o serviço avaliado.

Por outro lado, o fato de se estar considerando, nesse momento, apenas a perspectiva do prestador ou cliente interno e não considerando, também, a do cliente externo é, a princípio, uma limitação do presente trabalho.

\section{Metodologia}

O presente estudo é um trabalho exploratório e qualitativo e foi desenvolvido em duas etapas na área de abrangência da Secretaria Municipal de Saúde do município de Florianópolis (SC), que está estruturada em um nível central e cinco regionais de saúde (Centro, Leste, Sul, Norte e Continente) e conta com 48 Unidades Básicas de Saúde, uma Policlínica e dois Centros de Atenção Psicossocial. Quanto aos profissionais de saúde, o serviço possui 194 técnicos em enfermagem, 107 auxiliares em enfermagem, 150 enfermeiros, 220 médicos e 88 cirurgiões-dentistas.

A primeira etapa foi destinada à identificação dos indicadores que seriam utilizados. Inicialmente, realizou-se um levantamento dos Indicadores da Qualidade presentes na literatura para avaliação de serviços de saúde e que são tratados, indistintamente, por diversos autores como Tironi e colaboradores, 1991; Azevedo, 1993; Andreoni e Gualda, 1998; Bittar, 2001 e 2004; Marinho, 2001; Silveira, Santos e Costa, 2001; Kluck e colaboradores, 2002; Nepote, 2003; Aranha e Vieira, 2004; Valenzuela, 2005; Duarte e Ferreira, 2006.

Os Indicadores da Qualidade selecionados foram aqueles que poderiam ser utilizados para um serviço de odontologia, separados nas três categorias propostas por Donabedian (1988a, 1988b), ou seja, indicadores de Estrutura, de Processo e de Resultado.

Foi elaborado um questionário para ser aplicado aos cirurgiões-dentistas da rede municipal, aos coordenadores das cinco Regionais de Saúde e à coordenadora de Saúde Bucal do município que aceitassem participar da pesquisa. Os sujeitos da pesquisa deveriam escolher entre os indicadores apresentados em cada categoria aquele que consideravam o mais relevante para avaliar o 
serviço quanto à Estrutura, quanto ao Processo e quanto ao Resultado. Portanto, ao final da pesquisa de opinião seriam obtidos três indicadores para ser aplicados em uma Unidade Básica de Saúde do município.

O questionário foi pré-testado em um estudo piloto com profissionais de uma Secretaria Municipal de Saúde entrevistados pelos autores, mostrando-se adequado para o estudo pretendido.

A segunda etapa foi a efetiva utilização dos três indicadores apontados pelos sujeitos da pesquisa em uma das Unidades Básicas de Saúde do município. Para tanto, foi acompanhado um período de atendimento aos clientes na unidade. Os critérios adotados para a seleção da Unidade Básica de Saúde foram localização geográfica, ou seja, facilidade de acesso do pesquisador, oferta de maior número de serviços à população, atendimento em período integral e maior frequência de usuários por dia. Obteve-se liberação da Coordenação apenas para um dia, já que a presença constante de alguém estranho às atividades poderia interferir no serviço.

\section{Resultados e discussão}

\subsection{Primeira etapa}

O questionário aplicado continha vários indicadores distribuídos dentro das três categorias num total de 40 perguntas. Para cada indicador apresentado, os indivíduos pesquisados deveriam assinalar o grau de relevância do mesmo, de acordo com sua opinião, da seguinte forma (quadro 1):

$$
\text { Quadro } 1
$$

Exemplo de pergunta para aferir grau de relevância de indicador

2.1 Tempo de espera em fila ou lista de espera

( ) Irrelevante na avaliação da qualidade do serviço

( ) Pouco relevante na avaliação da qualidade do serviço

( ) Relevante na avaliação da qualidade do serviço

( ) Muito relevante na avaliação da qualidade do serviço

Fonte: Os autores. 
Foram apresentados 16 Indicadores de Estrutura:

1) tempo de espera em fila ou lista de espera;

2) tempo de espera entre a marcação da consulta e o atendimento;

3) número de mesas redondas semanais e o planejamento multidisciplinar;

4) localização geográfica;

5) número de profissionais cirurgiões-dentistas por habitante;

6) número de Centros de Saúde por população de referência;

7) número de consultas realizadas por profissional em um dia de trabalho;

8) horas de treinamento ou cursos fornecidos por instituição/cirurgião-dentista/ano;

9) uso de pessoal auxiliar;

10) taxa de absenteísmo;

11) turnover;

12) incentivos financeiros aos profissionais;

13) número de profissionais especialistas nas diversas áreas da odontologia;

14) gasto público em saúde per capita;

15) percentagem do gasto público em saúde dentro do gasto público total; e

16) número de equipamentos/consultórios e instrumentais disponíveis.

O questionário contava, ainda, com 15 Indicadores de Processo:

1) relação entre número de pacientes atendidos/funcionário;

2) relação entre número de atendimento/unidade de tempo;

3) tempo para realização de exames solicitados;

4) percentagem de exames repetidos;

5) número de prontuários incompletos;

6) número de prontuários com a primeira consulta em branco;

7) percentagem de diagnósticos errados;

8) percentagem de exames solicitados e não realizados; 
9) número de procedimentos errados;

10) número de consultas recebidas por paciente em um período de tempo;

11) razão entre o número de pessoas atendidas e a população de referência;

12) indicadores de resolutividade;

13) número de pacientes em assistência domiciliar;

14) número de visitas da ESB por paciente; e

15) cuidados com biossegurança.

Por último, possuía nove Indicadores de Resultado:

1) número de altas por período;

2) número de palestras, reuniões e atividades para comunidade;

3) indicadores de desperdício;

4) número de reclamações;

5) satisfação do usuário;

6) satisfação do prestador (cirurgião-dentista);

7) taxa de incidência ou prevalência de doenças;

8) número de acidentes de trabalho dos cirurgiões-dentistas da instituição; e

9) número de casos de doenças ocupacionais dos cirurgiões-dentistas que trabalham na instituição.

Foram disponibilizados 88 questionários, ou seja, o número de cirurgiões-dentistas do serviço; entretanto, após o término da coleta de dados, 62 questionários retornaram. A abstenção de $29,55 \%$ ocorreu devido ao fato de alguns profissionais não estarem disponíveis para a pesquisa por motivos diversos.

Dos questionários que retornaram, seis estavam incompletos e foram descartados. Portanto, foram considerados válidos 56 questionários dos 62 que retornaram, ou seja, $90,32 \%$, ou $63,64 \%$ dos 88 disponibilizados inicialmente, e que não apresentavam nenhuma questão em branco. As respostas foram tabuladas em planilha eletrônica de duas maneiras (quadros 2 e 3). 
Quadro 2

Utilizando as quatro alternativas do questionário

\begin{tabular}{|l|r|l|l|l|l|l|l|l|l|l|l|l|l|l|l|l|}
\hline $\begin{array}{l}\text { Indicadores de } \\
\text { Estrutura }\end{array}$ & 2.1 & & NOMES DOS & INDICAD & ORES (representados por números) & \\
\hline Irrelevante & 3 & & & & & & & & & & & & & & & \\
\hline Pouco Relevante & 10 & & & & & & & & & & & & & & \\
\hline Relevante & 26 & & & & & RESULTADOS DAS RES POSTAS & & & & \\
\hline Muito Relevante & 14 & & & & & & & & & & & & & & & \\
\hline Total de questionários & 53 & & & & & & & & & & & & & & & \\
\hline
\end{tabular}

Fonte: Os autores.

Quadro 3

Utilizando categorização, onde as opções irrelevante e pouco relevante foram transformadas em NÃO e as opções relevante e muito relevante, em SIM

\begin{tabular}{|c|c|c|c|c|c|c|c|c|c|c|c|c|c|c|}
\hline $\begin{array}{l}\text { Indicadores de } \\
\text { Estrutura }\end{array}$ & 2.1 & & NOMES & DOS & INDI & CAD & DORE & $=S(\mathrm{re}$ & pres & entad & dos $p$ & фr nú & imer & ps) \\
\hline Não & 13 & RES & ULTADOS & DAS & RESP & OSt & $\mathrm{AAS}=$ & $=|R R|$ & ELEW & ANTE & $+P C$ & juco & REL & LEVANTE \\
\hline Sim & 40 & $\mathrm{RE}$ & SULTADO & $S$ DAS & $S$ RES & $\mathrm{PO}$ & \$TAS & $=\mathrm{RE}$ & LEVA & ANTE & $+M L$ & पITO & RELE & EVANTE \\
\hline Total & 53 & & & & & & & & & & & & & \\
\hline
\end{tabular}

Fonte: Os autores

Esta categorização foi utilizada em virtude de, mesmo tendo sido testada em um estudo piloto, alguns participantes relatarem certa dificuldade em observar diferenças significativas entre as opções apresentadas. Portanto, para seleção do indicador mais votado em cada categoria se utilizou a tabela categorizada. No caso de empate entre dois indicadores, a utilização da tabela na íntegra seria o critério de desempate, onde aquele mais votado como muito relevante seria escolhido.

$\mathrm{Na}$ categoria Indicadores de Estrutura o indicador mais votado foi "gasto público per capita em saúde", com 56 votos. Entretanto, devido à dificuldade de acesso às informações ou aos dados necessários para calcular este indicador, foi selecionado o segundo colocado, "horas de treinamento ou cursos fornecidos pela instituição/cirurgiões-dentistas/ano”, com 55 votos. 
Para Indicadores de Processo houve empate entre quatro indicadores, ou seja, "tempo para realização de exames solicitados", "percentagem de diagnósticos errados", "número de procedimentos errados" e "cuidados com biossegurança", todos com 54 votos. Utilizando o critério de desempate, o indicador selecionado foi "cuidados com biossegurança", com 44 votos na opção muito relevante.

Quanto aos Indicadores de Resultado, também houve empate entre dois indicadores, ou seja, "satisfação do usuário" e "satisfação do prestador", com 55 votos. No entanto, o que apresentava maior número de votos como muito relevante, 36 votos, foi "satisfação do prestador", neste caso, o cirurgião-dentista.

Com os três Indicadores da Qualidade definidos, foi dado início à segunda etapa do estudo, ou seja, a aplicação, em que será possível avaliar suas exequibilidades.

\subsection{Segunda etapa}

Com os três indicadores já escolhidos e com a Unidade Básica de Saúde selecionada, definiu-se de que forma obter os dados para cada indicador e como explorá-los. Assim, os dados para o Indicador de Estrutura "horas de treinamento ou cursos fornecidos por instituição/cirurgião-dentista/ano" foram obtidos através de entrevista com a ex-coordenadora de Saúde Bucal do município.

Para avaliação do Indicador de Processo, "cuidados com biossegurança", utilizaram-se os critérios quanto às normas de biossegurança indicados na literatura científica, como os utilizados no manual de biossegurança elaborado pela Secretaria Estadual de Saúde do Estado de São Paulo (São Paulo, 2004). Esse manual apresenta um check list bastante completo dos itens de biossegurança a seram avaliados, sendo, também, mais didático que os demais manuais disponíveis.

No cálculo do Indicador de Resultado "satisfação do prestador" foi utilizada a versão resumida da Escala Brasileira de Avaliação da Satisfação (Satis-BR), proposta por Bandeira, Pitta e Marcier (2000). A versão resumida é composta por 32 questões quantitativas que avaliam o nível de satisfação do prestador com o serviço, através da média aritmética das questões, em que quanto mais perto de cinco for este valor maior será a satisfação do mesmo. A escala pode avaliar a satisfação global ou por grupos, sendo um conjunto de questões responsável por avaliar a satisfação do prestador com a qualidade do serviço prestado ao paciente, outro por avaliar a satisfação com relação à participação do prestador no serviço, um para avaliar a satisfação com relação às 
condições gerais de trabalho ou, ainda, a satisfação quanto ao relacionamento com colegas e superiores.

Após esta definição, os três indicadores foram aplicados na Unidade Básica de Saúde predefinida. No dia da coleta de dados estavam presentes na unidade três cirurgiões-dentista, dos quais dois aceitaram participar da pesquisa.

O primeiro ponto a ser discutido com relação à aplicação dos indicadores é sua exequibilidade, ou seja, é ou não possível utilizar o indicador na instituição? E como informação secundária ou complementar, avaliar se há ou não qualidade em cada um destes indicadores na Unidade Básica de Saúde. Para Donabedian (1988a), toda avaliação, sendo os indicadores um tipo de avaliação, para ser realizada depende do acesso a informações precisas e adequadas. Essas informações podem ser análise documental, entrevistas ou questionários com os envolvidos na atividade ou, ainda, através de observações diretas ou indiretas (fotos, filmagens etc.) do contexto a ser avaliado. As informações para aplicação dos indicadores deste estudo foram obtidas através de questionários, entrevistas e observações diretas.

Para o Indicador de Estrutura, "horas de treinamento ou cursos fornecidos por instituição/cirurgião-dentista/ano", foi analisado o período de um ano, compreendido entre dezembro de 2005 e dezembro de 2006, pois a instituição não dispunha de dados mais recentes registrados. Neste período, alguns dos cursos, palestras ou treinamentos ofertados pela instituição aos profissionais cirurgiões-dentistas da rede foram os seguintes (quadro 4).

\section{Quadro 4}

Quadro de cursos, palestras e treinamentos disponibilizados pela Secretaria Municipal de Saúde do município de Florianópolis

\begin{tabular}{|c|c|c|}
\hline DATA & CAPACITAÇÃO & CARGA HORÁRIA \\
\hline \multirow{2}{*}{ Mar. 2006} & Tema: Promoção em saúde bucal & - \\
\hline & Tema: Programa Saúde da Família este desconhecido & - \\
\hline \multirow{2}{*}{ Abr. 2006} & Tema: Equidade e Integralidade & - \\
\hline & Tema: O Programa Saúde da Família em saúde bucal & - \\
\hline Maio 2006 & Curso de geriatria e odontogeriatria & 40 horas \\
\hline Nov. 2006 & Tema: Biossegurança & - \\
\hline Dez. 2006 & $\begin{array}{l}\text { Tema: Tabagismo - uma abordagem para o tratamento do } \\
\text { fumante }\end{array}$ & - \\
\hline
\end{tabular}

Fonte: Secretaria Municipal de Saúde do município de Florianópolis. 
Além destes cursos e de treinamento de capacitação, constam da programação da instituição reuniões mensais dos cirurgiões-dentistas da rede com a Coordenação de Saúde Bucal, com cerca de quatro horas cada. Estas reuniões são realizadas com o intuito de discutir e apresentar as experiências de cada regional de saúde de forma que as experiências positivas de uma unidade possam servir como parâmetro para outra, ou seja, o melhor que cada área faz e, assim, buscar a melhoria contínua.

As atividades de benchmarking podem ser externas, quando o padrão é fixado por outra instituição do ramo, ou internas, quando o padrão é posto pela própria instituição que almeja melhorar suas próprias metas (Paladini, 2000, 2002). É o segundo que está sendo aplicado pela Secretaria Municipal de Saúde de Florianópolis para conseguir a melhoria de seus processos nas diversas Unidades Básicas de Saúde, no momento em que discute o que é feito de melhor em determinada unidade para aplicar em outra.

$\mathrm{O}$ acesso a dados precisos é extremamente limitado, pouco organizado e adequado, condição fundamental para a utilização de qualquer indicador (Paladini, 2000; Cecílio, 2002; Mello e Camargo, 1998). Assim, o Indicador de Estrutura, "horas de treinamento ou cursos fornecidos por instituição/cirurgião-dentista/ano", não se mostrou exequível nesse momento.

Não há na instituição uma agenda oficial dos cursos ou treinamentos fornecidos por ela, nem um controle exato de quais profissionais participaram de quais eventos, o que dificulta a aplicação deste indicador, apontando não haver qualidade neste quesito, pois não há planejamento prévio de quando, de que forma e quem participará efetivamente dos treinamentos. Segundo Paladini $(2000,2002)$, planejamento é fundamental para qualidade, pois planejar significa agir baseado em fatos concretos e objetivos e não de forma imprevista ou intuitiva.

Não se buscou utilizar um terceiro indicador, pois esse não iria refletir o resultado das respostas da primeira etapa, tendo em vista que quatro indicadores obtiveram a mesma quantidade de escolhas, ou seja, 54 para cada um.

Sobre o segundo indicador, Indicador de Processo, "cuidados com biossegurança", aplicado na Unidade Básica de Saúde selecionada, foi levado em consideração o padrão de biossegurança definido pelo estado de São Paulo em seu manual sobre qualidade e biossegurança.

O manual apresenta para avaliação itens como: forma adequada da lavagem das mãos, uso de equipamentos de proteção individual, cuidados com limpeza e desinfecção do ambiente de atendimento bem como do equipo, cuidado e manuseio dos instrumentos perfurocortantes e como proceder no caso de acidentes com os mesmos. Ainda: cuidados com processamento e esterili- 
zação dos materiais, bem como desinfecção daqueles que não podem ser esterilizados por meio físico, cuidados com radiação, cuidados com manipulação de materiais que trazem risco químico como o mercúrio e risco ergonômico (ruído, postura, iluminação etc.).

Ao tratar de biossegurança, não é possível aceitar meio certo; assim, os itens que apresentavam algum ponto distinto do preconizado foram considerados incompatíveis. Dos 15 itens de biossegurança analisados pelo manual, o dentista 1 estava compatível com seis itens (uso de luvas, uso de máscaras, uso de gorro, como proceder em acidentes perfurocortantes, risco ergonômico e cuidados com a equipe) e o dentista 2 com cinco itens (uso de luvas, máscaras e gorro, como proceder em acidentes perfurocortantes e cuidados com a equipe).

A partir destes resultados, pode-se dizer que este indicador é exequível, através de entrevistas e observação, tomando como referência o manual de biossegurança do estado de São Paulo, sendo plenamente possível obter os dados necessários, de forma clara e precisa. Quanto à qualidade, considerando a afirmação da Agência de Vigilância Sanitária (2005), para a qual tratar de biossegurança é analisar os riscos a que está sujeita a vida, não sendo possível aceitar meio certo quando a questão é proteger a saúde individual e coletiva. Assim, apenas cinco ou seis itens em concordância é um valor muito baixo, não apresentando esta Unidade Básica de Saúde qualidade quanto a esse indicador.

Com relação ao último indicador, Indicador de Resultados, "satisfação do prestador", de acordo com a escala Satis-BR, quanto mais próximo de cinco for o valor obtido na média aritmética do total de questões ou por grupos, maior é a satisfação do prestador. Baseado na escala de um a cinco, estabeleceu-se a seguinte relação para posterior avaliação do grau de satisfação do prestador.

Figura

Escala para avaliação da pontuação sobre a satisfação do prestador

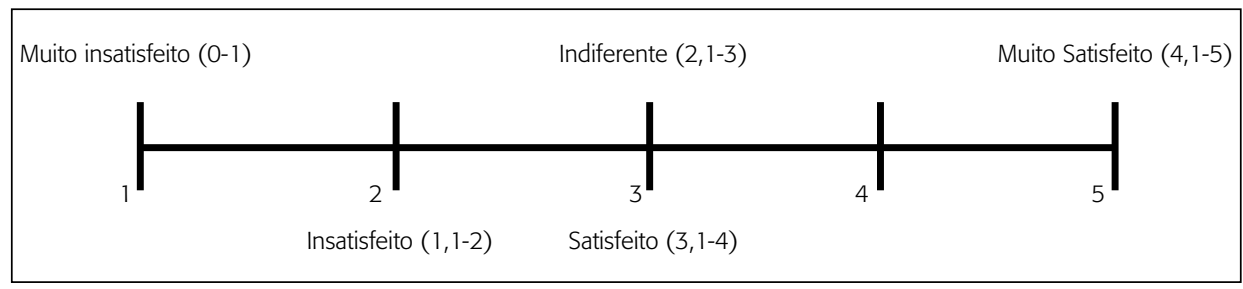

Fonte: Os autores. 
A média aritmética dos dois profissionais indicou os seguintes resultados (quadro 5).

Quadro 5

Resultados para o indicador "satisfação do prestador"

\begin{tabular}{|l|c|c|}
\hline & CIRURGIÃO-DENTISTA 1 & CIRURGIÃO-DENTISTA 2 \\
\hline Satisfação Global & 3,4 & 3,9 \\
\hline $\begin{array}{l}\text { Satisfação Quanto à Qualidade dos } \\
\text { Serviços Oferecidos ao Cliente }\end{array}$ & 3,7 & 3,8 \\
\hline $\begin{array}{l}\text { Satisfação Quanto à Participação da } \\
\text { Equipe no Serviço }\end{array}$ & 3,9 & 4,0 \\
\hline $\begin{array}{l}\text { Satisfação Quanto às Condições Gerais de } \\
\text { Trabalho }\end{array}$ & 2,8 & 4,0 \\
\hline $\begin{array}{l}\text { Satisfação Quanto à Relação com a } \\
\text { Equipe de Trabalho }\end{array}$ & 3,0 & 3,5 \\
\hline
\end{tabular}

Fonte: Os autores.

De acordo com os resultados, pode-se identificar que, de uma forma geral, ambos apresentam-se satisfeitos com a instituição. Porém, o cirurgiãodentista 1 encontra-se no nível indiferente no item condições gerais de trabalho, quando avaliada a satisfação pelos quatro grandes grupos, o que pressupõe certa insatisfação. Este grupo inclui a satisfação do prestador a respeito dos seguintes aspectos: a) as condições gerais das instalações; b) o salário e os benefícios recebidos; c) o conforto e a aparência do serviço; d) as medidas de segurança e de confidencialidade em vigor no mesmo; e e) o clima do ambiente de trabalho. Por outro lado, o cirurgião-dentista 2 encontra-se satisfeito também neste item.

Deve-se salientar que há uma diferença de tempo de casa entre os dois: o profissional 1 está na instituição há cinco anos e o profissional 2, há apenas oito meses. Por outro lado, o cirurgião-dentista 2 também não é contratado efetivo da instituição, sendo residente do Programa de Residência em Saúde da Família, do qual a instituição faz parte juntamente com a Universidade Federal de Santa Catarina. Esses dois fatores podem ter colaborado para a grande divergência de opinião entre os dois neste aspecto.

Resultado semelhante ocorreu no estudo realizado por Rebouças, Legay e Abelha (2007), que realizaram uma pesquisa sobre a satisfação em profissionais de saúde mental. Entre esses profissionais, aqueles que não apresentavam forte vínculo profissional com a instituição não responderam à pesquisa, possi- 
velmente por se sentirem constrangidos ou amedrontados com a possibilidade de serem identificados no estudo e com isso sofrerem represálias por parte dos gestores. Os autores encontraram, ainda, resultados semelhantes quanto ao nível de satisfação geral intermediário, muito próximo dos 3,29 encontrados no presente estudo, sendo o item condições gerais de trabalho, também, o que apresentava maior insatisfação por parte da equipe.

Os resultados do trabalho coincidem com os de Nicolielo e Bastos (2002), que desenvolveram um estudo para avaliar a satisfação de profissionais cirurgiões-dentistas com seu trabalho. Os resultados encontrados pelos autores estão de acordo com os do presente estudo: o maior índice de insatisfação, para ambos os estudos, estava no baixo rendimento ou honorários recebidos pelo trabalho realizado, salientando que os piores índices de insatisfação para o cirurgião-dentista 1 estão no grupo condições de trabalho, que inclui a questão salarial e de benefícios.

Quanto à exequibilidade, este indicador é passível de ser utilizado na instituição a partir do questionário selecionado para avaliação. Por outro lado, quanto à qualidade, apenas um dos itens foi considerado indiferente.

\section{Conclusões}

Baseado no estudo realizado, pode-se concluir que os três indicadores aplicados são passíveis de ser utilizados nas Unidades Básicas de Saúde da Secretaria Municipal de Saúde do município de Florianópolis, desde que se tenha acesso aos dados de forma clara e precisa. Assim, mesmo o indicador "horas de treinamento ou cursos fornecidos por instituição/cirurgião-dentista/ano", que não pôde ser aplicado adequadamente por não haver disponibilidade dos dados de forma clara, precisa e organizada, tornar-se-ia exequível como os demais, se houver maior controle destas informações.

A partir dos resultados encontrados, é possível dizer, também, que apresentar qualidade em uma das três categorias não é necessariamente sinônimo de apresentá-la nos demais aspectos, já que, mesmo o serviço não apresentando um resultado positivo quanto à biossegurança e ao treinamento, no entanto, indicou resultado positivo quanto à satisfação do prestador. Assim, os indicadores se complementam, mas não são, necessariamente, coincidentes.

Conclui-se, ainda, que os indicadores estão compatíveis com as características esperadas para um bom indicador, de acordo com Bittar (2001):

1) Objetividade: já que expressam de forma simples e direta a situação a que se referem; 
2) Simplicidade: fáceis de buscar os dados, calculá-los e analisá-los;

3) Validade: capazes de identificar as situações que devem ser melhoradas;

4) Sensibilidade: capazes de identificar todos os problemas existentes com detalhes; e

5) Baixo custo para sua execução: o que viabiliza sua aplicação.

Os resultados observados permitem fazer uma avaliação inicial quanto à qualidade do serviço de odontologia ofertado pela Secretaria Municipal de Saúde do município de Florianópolis aos usuários da unidade analisada. Entretanto, não se devem generalizar os resultados obtidos para as demais Unidades Básicas de Saúde, pois cada uma pode apresentar uma realidade de trabalho diferente.

Portanto, o estudo cumpre seu objetivo de avaliar a qualidade dos serviços públicos de odontologia oferecidos por uma Unidade Básica de Saúde de um município, através do uso de Indicadores da Qualidade, mas não permite aferir a qualidade efetiva do serviço de odontologia do município de Florianópolis por aplicá-los em apenas uma unidade.

Por outro lado, recomenda-se que a organização estenda o uso de indicadores para outras Unidades Básicas de Saúde do município com o objetivo de conhecer a real qualidade do serviço prestado como um todo e apontar melhorias.

\section{Referências}

ANDREONI, S.; GUALDA, D.M.R. Padrões e critérios como indicadores no processo de Controle de Qualidade Assistencial: relato de experiência. Nursing, São Paulo, v. 1, n. 5, p. 13-17, out. 1998.

AGÊNCIA DE VIGILÂNCIA SANITÁRIA (ANVISA). Informes técnicos institucionais: biossegurança. Revista de Saúde Pública, São Paulo, v. 39, n. 6, p. 989-991, 2005.

ARANHA, G.T.C.; VIEIRA, R.W. Estudo de um dos indicadores do custo da qualidade: o desperdício. Revista de Administração em Saúde, São Paulo, v. 6, n. 23, p. 1-13, abr./jun. 2004.

AZEVEDO, A.C. Indicadores da qualidade e produtividade em serviço de saúde. Revista Indicadores da Qualidade e Produtividade, São Paulo, v. 1, n. 1, p. 47-55, fev. 1993. 
BANDEIRA, M.; PITTA, A.M.F.; MARCIER, C. Escalas brasileiras de avaliação da satisfação (Satis-BR) e da sobrecarga (Impacto-BR) da equipe técnica em serviços de saúde mental. Jornal Brasileiro de Psiquiatria, Rio de Janeiro, v. 49, n. 1, p. $105-115,2000$.

BITTAR, O.J.N.V. Indicadores de qualidade e quantidade em saúde. Revista de Administração em Saúde, São Paulo, v. 3, n. 12, p. 21-28, jul./set. 2001.

. Indicadores de qualidade e quantidade em saúde: parte II. Revista de Administração em Saúde, São Paulo, v. 6, n. 22, p. 15-18, jan./mar. 2004.

CECÍLIO, L.C. de O. O pagamento de incentivos financeiros para os funcionários como parte da política da qualificação da assistência de um hospital público, Volta Redonda, Rio de Janeiro, Brasil. Cadernos de Saúde Pública, Rio de Janeiro, v. 18, n. 6, p. 1655-1663, nov./dez. 2002.

DONABEDIAN, A. The quality of care: how can it be assessed? Journal of American Medical Association, New York, v. 260, n. 12, p. 1743-1748, Sept. 1988a.

. Quality assessment and assurance: unity and purpose, diversity of means. Inquirity, Ribeirão Preto, v. 25, n. 1, p. 179-192, Spring 1988b.

DUARTE, I.; FERREIRA, D.P. Uso de indicadores na gestão de um centro cirúrgico. Revista de Administração em Saúde, São Paulo, v. 8, n. 31, p. 63-70, abr./jun. 2006.

FADEL, M.A.V.; REGIS FILHO, G.I. Percepção da qualidade em serviços públicos de saúde: um estudo de caso. Revista de Administração Pública, Rio de Janeiro, v. 43, n. 1, p. 7-22, jan./fev. 2009.

KLUCK, M. et al. A gestão da qualidade assistencial do Hospital de Clínica de Porto Alegre: implantação e validação de indicadores. Revista de Administração em Saúde, São Paulo, v. 4, n. 16, p. 27-32, jul./set. 2002.

MALIK, A.M.; SCHIESARI, L.M.C. Qualidade na gestão local de serviços e ações de saúde. São Paulo: Fundação Petrópolis, 1998.

MARINHO, N. Avaliação da eficiência técnica nos serviços de saúde dos municípios do estado do Rio de Janeiro. Brasília: Ipea, nov. 2001. (Texto para discussão n. 842)

MELLO, J.B.; CAMARGO, M.O. Práticas e conceitos: normas ISO nas áreas médicohospitalar e laboratorial. São Paulo: Best Seller, 1998.

MIGUEL, P.A.C.; SALOMI, G.E. Uma revisão dos modelos para medição da qualidade em serviços. Revista Produção, São Paulo, v. 14, n. 1, 2004.

MILAN, G.S.; TONI, D. de; BARAZETTI, L. Configuração e organização de imagens de serviços: um estudo exploratório. Revista Produção, São Paulo, v. 15, n. 1, p. 60-73, jan./abr. 2005. 
NEPOTE, M.H. A. Análise do desempenho das atividades no centro cirúrgico através de indicadores. Revista de Administração em Saúde, São Paulo, v. 5, n. 21, p. 21-30, out./dez. 2003.

NICOLIELO, J.; BASTOS, J.R. de M. Satisfação profissional do cirurgião-dentista conforme tempo de formado. Revista da Faculdade de Odontologia, Bauru, v. 10, n. 2, p. 69-74, 2002.

PALADINI, E.P. Gestão da qualidade: teoria e prática. São Paulo: Atlas, 2000. . Avaliação estratégica da qualidade. São Paulo: Atlas, 2002.

REBOUÇAS, D.; LEGAY, L.F.; ABELHA, L. Satisfação com o trabalho e o impacto causado nos profissionais de serviço de saúde mental. Revista de Saúde Pública, São Paulo, v. 41, n. 2, p. 244-250, 2007.

SÃO PAULO. Governo do Estado de São Paulo. Cadernos de saúde bucal: qualidade e resolutividade na atenção básica — recomendações de biossegurança. Secretaria de Estado da Saúde, 2004. Disponível em: <ftp://ftp.saude.sp.gov.br/ftpsessp/ novo_site/saude_bucal/cadernos/biosseguranca.pdf>. Acesso em: 25 set. 2007.

SILVEIRA, D.S. da; SANTOS, I.S. dos; COSTA, J.S.D. da. Atenção pré-natal na rede básica: uma avaliação da estrutura e do processo. Cadernos de Saúde Pública, Rio de Janeiro, v. 17, n. 1, p. 131-139, jan./fev. 2001.

SOÁREZ, P.C. de; PADOVAN, J.L.; CICONELLI, R.M. Indicadores de saúde no Brasil: um processo em construção. Revista de Administração em Saúde, São Paulo, v. 7, n. 27, p. 57-64, abr./jun. 2005.

TENGAN, C. Abordagem teórica e aplicação de um método de qualidade em serviço público odontológico. Tese (doutorado) - Programa de Pós-Graduação em Odontologia, Faculdade de Odontologia de Piracicaba, Universidade Estadual de Campinas, Piracicaba, 2008.

TIRONI, L.F. et al. Critérios para geração de indicadores da qualidade e produtividade no serviço público. Brasília: Ipea, out. 1991. (Texto para discussão n. 238)

TONTINI, G.; SANT'ANA, A.J. Interação de atributos atrativos e obrigatórios de um serviço na satisfação do cliente. Revista Produção, São Paulo, v. 18, n. 1, p. 112-125, jan./abr. 2008.

; SILVEIRA, A. Identification of satisfaction attributes using competitive analysis of the improvement gap. International Journal of Operations \& Production Management, v. 27, n. 5, p. 482-500, 2007.

VALENZUELA, M.T. Indicadores de salud: características, uso y exemplos. Ciência \& Trabajo, Providência, año 7, n. 17, p. 118-122, jul./set. 2005. 